\title{
Chondroprotective Effect of Zerumbone on Monosodium Iodoacetate Induced Osteoarthritis in Rats
}

\begin{abstract}
The objective of this investigation was to evaluate chondroprotective effect of zerumbone, a purified compound of Zingiber zerumbet Smith against monosodium iodoacetate (MIA) induced knee osteoarthritis (OA) in the rat. The effect on the articular cartilage was examined and compared with celecoxib (Celebrex $\left.{ }^{\circledR}\right)$, a Non-Steroidal Anti-Inflammatory Drug (NSAID). Forty adult male Sprague Dawley rats were divided into four groups $(\mathrm{n}=10$ for each). All animals were injected with MIA intraarticularly in their right knee joints to induce OA. Rats from first and second groups were treated with zerumbone in a same dose but with two different concentrations. Rats in the third group were treated with celecoxib and served as positive control whereas the fourth group were treated with corn oil and served as negative control. Evaluation of OA changes in the knees was assessed with the aid of both radiography and histopathology score. Macroscopic as well as microscopic examinations revealed curative effect of zerumbone in a dose dependent manner on the osteoarthritic knee joints. Apart from this, our data also revealed very poor anti-OA property of celecoxib. We concluded that oral administration of zerumbone in a dose of $2 \mathrm{~mL} \mathrm{kg-1} \mathrm{b.wt.} \mathrm{of} 0.4 \% \mathrm{w} / \mathrm{v}$ diluted with corn oil for a period of 4 weeks has some chondroprotective effects.
\end{abstract}

Keyword: Osteoarthritic, Zerumbone, monosodium iodoacetate, cartilage, medicinal plants, rat 